\title{
Avaliação radiológica do espaço articular na artrose do quadril: estudo comparativo em decúbito e ortostatismo"
}

\author{
Radiologic evaluation of joint space in hip arthrosis: \\ comparative study in decubitus and upright
}

Gustavo Fornari Vanni' ${ }^{1}$, Jules Michel Stucky², Carlos Roberto SchwarstmanN³

\section{RESUMO}

Objetivo: Comparar as alterações do espaço articular em pacientes com osteoartrose do quadril, por meio de estudo radiográfico que inclui as posições em decúbito dorsal e ortostatismo monopodálico. Métodos: A amostra do presente estudo consta de 74 radiografias. Metade das radiografias foram realizadas com os pacientes em decúbito dorsal e a outra metade com os pacientes em ortostatismo monopodálico. Uma régua milimetrada e uma lente de aumento foram usadas para realizar as medições dos quadris. $O$ espaço entre o acetábulo e a cabeça femoral (EACF) foi medido a partir de radiografias centradas no quadril. Resultados: Com-

* Trabalho realizado no Complexo Hospitalar da Santa Casa de Porto Alegre (RS), Brasil.

1. Residente do terceiro ano do Serviço de Ortopedia e Traumatologia do Complexo Hospitalar da Santa Casa de Porto Alegre (RS), Brasil.

2. Residente do primeiro ano do Serviço de Ortopedia e Traumatologia do Complexo Hospitalar da Santa Casa de Porto Alegre (RS), Brasil.

3. Professor Titular de Ortopedia da Fundação Faculdade Federal de Ciências Médicas de Porto Alegre - FFCMPA. Chefe do Serviço de Ortopedia e Traumatologia do Complexo Hospitalar da Santa Casa de Porto Alegre (RS), Brasil.

Endereço para correspondência: Rua Euclides da Cunha, 493, Bairro Partenon - 90620-220 - Porto Alegre (RS), Brasil. Tel./fax: (51) 3336-0382. E-mail: gfvanni@terra.com.br

Recebido em 20/7/08. Aprovado para publicação em 8/10/08.

Copyright RBO2008 parando-se 74 radiografias com osteoartrose do quadril obtidas em decúbito dorsal e ortostatismo monopodálico conclui-se que existe diferença estatisticamente significativa entre as posições estudadas respectivamente de 1,47 para $1,12 \mathrm{~mm}$ ( $p<$ $0,0001)$. A correlação foi de 0,952 com $p<0,0001$. $O$ teste "t-Student" pareado para a amostra decúbito dorsal e ortostatismo revelou intervalo de confiança de 0,2671 a 0,4302 com $p<0,0001$ bicaudal. Conclusão: As radiografias mensuradas em ortostatismo com apoio monopodálico avaliam melhor o verdadeiro espaço articular e o grau de artrose.

Descritores - Osteoartrite/radiografia; Articulação do quadril/radiografia; Radiografia/métodos

\section{ABSTRACT}

Objective: To compare changes in the joint space of patients with hip osteoarthrosis using radiographic studies that include positions lying on the back and standing on one foot. Methods: The sample of this study includes $74 X$-rays. Half of them were made with the patients lying on their backs, and in the other half, the patients were standing on one foot. A ruler with millimeter markings and a magnifying lens were used to measure the hips. The space between the acetabulum and the femoral head (EACF) was measured using $X$-rays centered on the hip. Results: Comparing $74 X$-rays with hip osteoarthrosis taken with the patient lying on the back and standing on one foot, conclusion is that there is a statistically 
significant difference between the positions studied, from 1.47 to $1.12 \mathrm{~mm}$, respectively $(p<0.0001)$. The correlation was 0.952 with $p<0.0001$. The paired $t$ student test for the lying on the back sample and for the upright sample showed a confidence interval of 0.2671 to 0,4302 , with $p<0.0001$, double tailed. Conclusion: X-rays measured in the upright position standing on a single foot provide a better evaluation of the joint space and of the degree of arthrosis.

Keywords - Osteoarthritis/radiography; Hip joint/ radiography; Radiography/methods

\section{INTRODUÇÃO}

A osteoartrose é uma doença da cartilagem articular e muitos fatores podem afetá-la. O processo atinge inicialmente a cartilagem e, após, o osso subcondral. Clinicamente, ela é caracterizada por dor e limitação funcional. Sem dúvida, o exame complementar mais importante para o diagnóstico é a radiografia. $\mathrm{O}$ estudo radiográfico também pode quantificar a osteoartrose. Ele preenche os critérios de facilidade, disponibilidade e custo $^{(1)}$. De acordo com Altman et al, a amplitude do espaço articular parece ser o critério mais importante para avaliar a progressão da osteoartrose do ponto de vista radiológico ${ }^{(2)}$. A esclerose subcondral e a presença de osteófitos não têm boa correlação com o comportamento clínico da doença. A posição do paciente no estudo radiográfico parece ter significativa importância na osteoartrose do joelho. Entretanto, nos casos de osteoartrose do quadril ainda não existe consenso $^{(3)}$. Na maioria, das vezes, o estudo radiográfico do quadril é realizado com o paciente em decúbito dorsal.

O presente estudo tem como objetivo comparar, utilizando o estudo radiográfico, a alteração do espaço articular em pacientes com osteoartrose do quadril avaliada nas posições de decúbito dorsal e ortostatismo monopodálico.

\section{MÉTODOS}

A amostra do presente estudo consta de 74 radiografias. Metade das radiografias foram realizadas com os pacientes em decúbito dorsal e a outra metade, com os pacientes em ortostatismo monopodálico. Foram avaliados 28 pacientes, sendo nove radiografias bilaterais e 19 unilaterais (tabela 1). O sexo masculino correspondia a 19 pacientes $(67,9 \%)$. O quadril direito foi avaliado em 24 exames radiográficos $(64,9 \%$ dos exames). Prótese contralateral foi encontrada em três pacientes. A média de idade dos pacientes foi de 56,07 com desvio-padrão de $\pm 12,487$ e com intervalo de 20 a 77 anos.

TABELA 1

Resultados obtidos com as radiografias realizadas em decúbito dorsal e ortostatismo

\begin{tabular}{lccc}
\hline \multicolumn{1}{c}{ Grupos } & $\begin{array}{c}\text { Média } \\
\text { (milímetros) }\end{array}$ & Casos & $\begin{array}{c}\text { Desvio } \\
\text { padrão }\end{array}$ \\
\hline Decúbito & 1,47 & 37 & 0,786 \\
Ortostatismo & 1,12 & 37 & 0,788 \\
\hline
\end{tabular}

Os pacientes estavam registrados no Ambulatório do Quadril do Complexo Hospitalar da Santa Casa de Porto Alegre e vinham sendo tratados para osteoartrose do quadril. Foram incluídos no trabalho somente pacientes com coxartrose de causa idiopática e sem outras afecções que pudessem estar relacionadas com a causa da artrose. Os exames radiográficos do quadril foram realizados no Serviço de Radiologia da Santa Casa de Porto Alegre, na mesma sala radiológica, pelo mesmo técnico e todos acompanhados pelo mesmo médico. As radiografias foram feitas na posição de decúbito dorsal e na ortostástica. A primeira radiografia do quadril a ser estudada foi tirada com o paciente em decúbito dorsal e sem que fosse exercida tração sobre a articulação. Na segunda posição, o paciente foi colocado em ortostatismo monopodálico com o joelho do membro inferior contralateral ao avaliado fletido em $90^{\circ}$ (figuras 1 e 2 ). As radiografias foram realizadas com distância de $110 \mathrm{~cm}$ entre o filme e a fonte do raio-X. A radiografia do quadril foi feita com a fonte do raio-X posicionada no centro da articulação e com o membro inferior com $25^{\circ}$ de rotação interna, nas duas posições.

Uma régua milimetrada e uma lente de aumento foram usadas para realizar as medições dos quadris. Fo- 


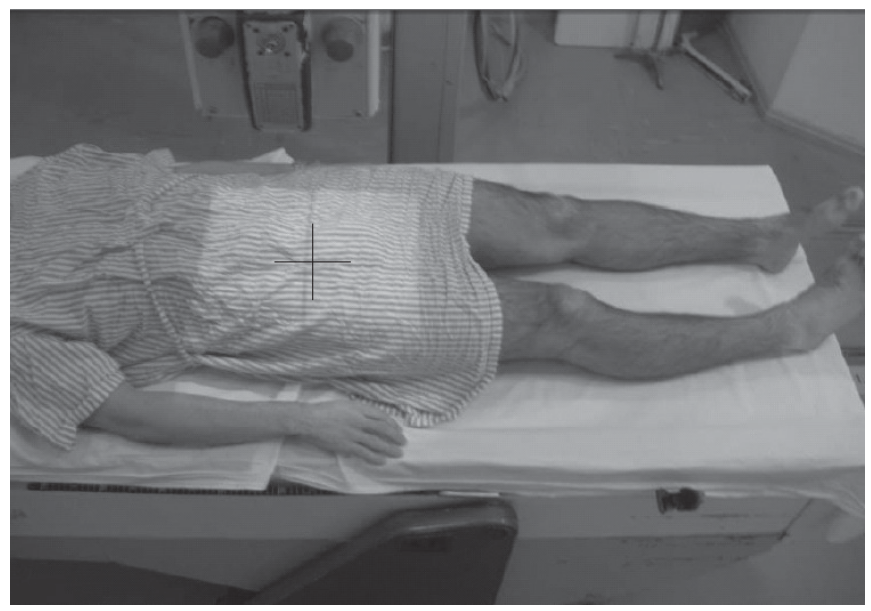

Figura 1 - Método de obtenção da radiografia do quadril em decúbito dorsal

ram usadas as "linhas de mouse" para verificar a esfericidade da cabeça femoral. O espaço entre o acetábulo e a cabeça femoral (EACF) foi medido a partir de radiografias centradas no quadril. O centro do quadril foi determinado baseado no rebordo lateral do quadril e na lágrima. Uma linha foi traçada entre esses dois pontos de referência (C). Do meio dessa linha, uma nova linha foi traçada até a segunda vértebra sacral (S2). Isso delimitava um quadrante superior responsável pela absorção da maior parte da carga que atravessa a articulação. O quadrante superior foi dividido em três porções articulares de igual tamanho. A medida da alteração do espaço articular foi realizada no meio da porção central, tanto em decúbito dorsal como em apoio monopodálico (figuras 3 e 4 ).

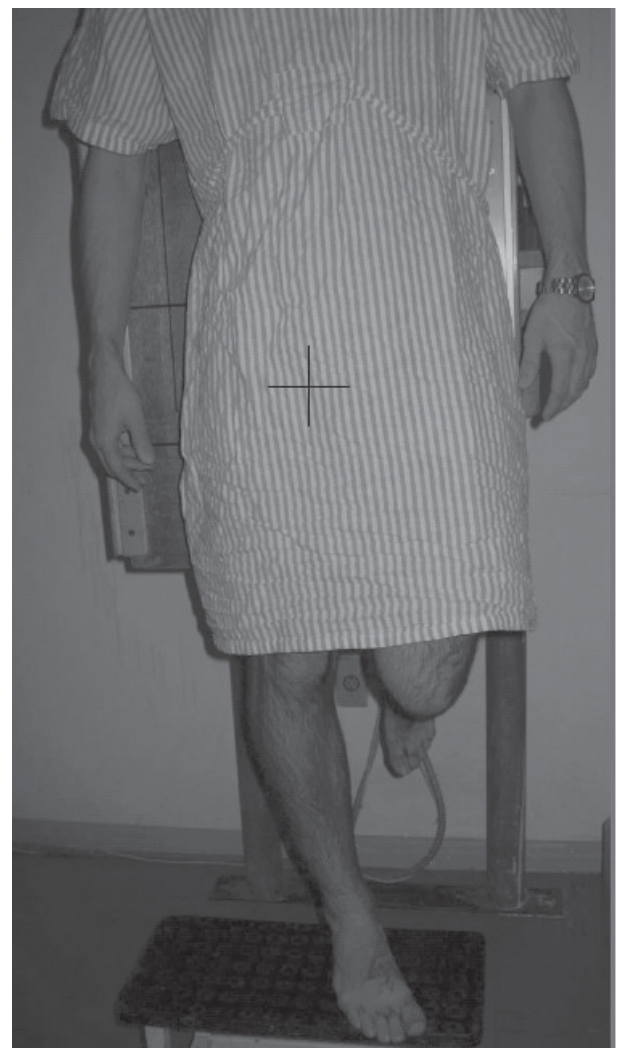

Figura 2 - Método de obtenção da radiografia do quadril em apoio monopodálico

Foi realizada análise descritiva dos dados demográficos da população do estudo com média, desvio-padrão e intervalo de amostra. As radiografias foram comparadas usando o teste "t-Student" pareado.

\section{RESULTADOS}

Os dados provenientes de nossa amostra de 74 radiografias demonstraram existir uma média de 1,47mm

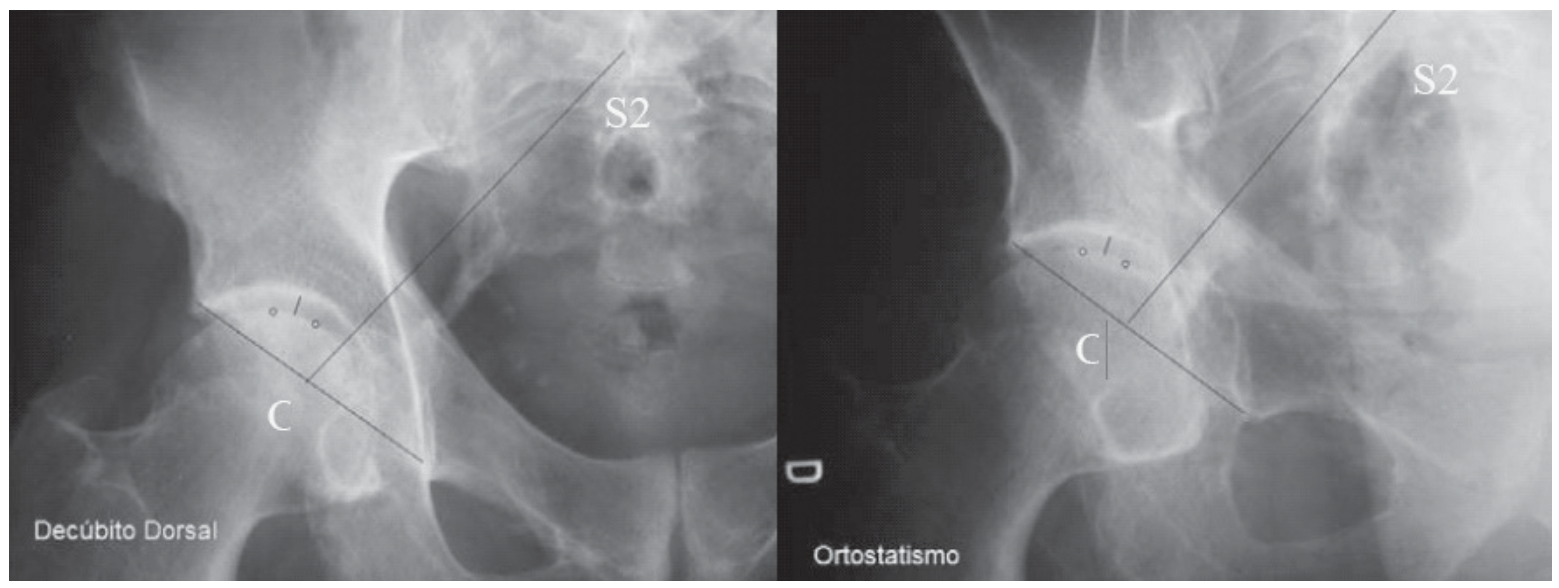

Figura 3 - Método aplicado em um caso - observar a diminuição do espaço articular comparando o decúbito dorsal e o ortostatismo 
Figura 4 - Método aplicado em segundo caso - observar a diminuição do espaço articular comparando o decúbito dorsal e o ortostatismo

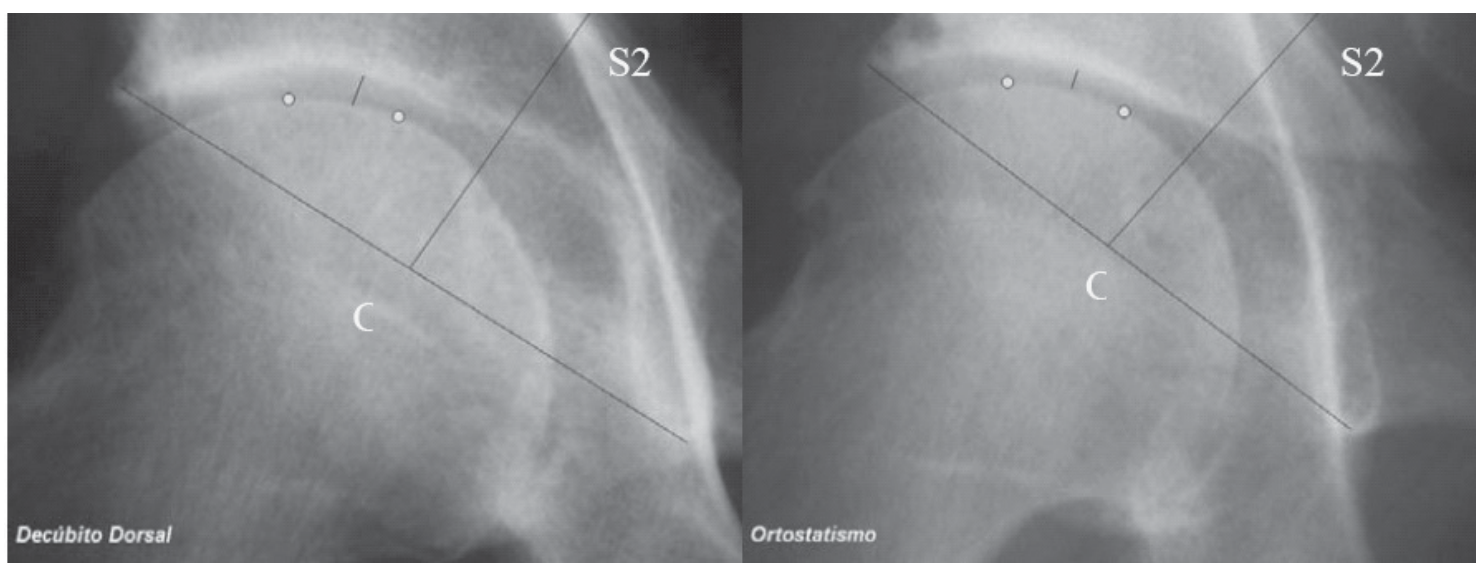

de distância na radiografia em decúbito dorsal do espaço entre o quadril e a cabeça femoral na sua maior distância, com intervalo de no mínimo 0,20 e no máximo de 2,90mm e desvio-padrão equivalente a 0,786. Já na posição de ortostatismo, as radiografias apresentaram uma média de $1,12 \mathrm{~mm}$ do mesmo espaço, com intervalo de no mínimo 0,10 e no máximo de $2,80 \mathrm{~mm}$ e desvio-padrão equivalente a 0,788 (tabela $1)$.

A amostra de pacientes apresentou correlação significativa em relação ao decúbito dorsal e ao ortostatismo. A correlação foi de $0,952 \operatorname{com} p<0,0001$. O teste "t-Student" pareado para a amostra decúbito dorsal e ortostatismo revelou intervalo de confiança de 0,267 a 0,430, com $\mathrm{p}<0,0001$ bicaudal (gráfico 1).

\section{DISCUSSÃO}

A artrose é caracterizada radiograficamente por: cistos subcondrais, diminuição do espaço articular, esclerose subcondral, osteófitos e subluxação articular. De todos os sinais radiológicos, a diminuição do espaço articular é aceita como fator mais relevante para avaliar a progressão da osteoartrose ${ }^{(3-5)}$. Entretanto, na maioria das vezes, os ortopedistas avaliam o espaço articular em radiografias em decúbito dorsal, o que pode falsear a real diminuição do mesmo, pois a articulação não está sendo avaliada durante a sua função, isto é, em ortostatismo monopodálico.

A deformação da cartilagem articular durante a carga é relacionada com a integridade das proteoglicanas e da matriz ${ }^{(3)}$. A perda de proteoglicanas altera as pro-

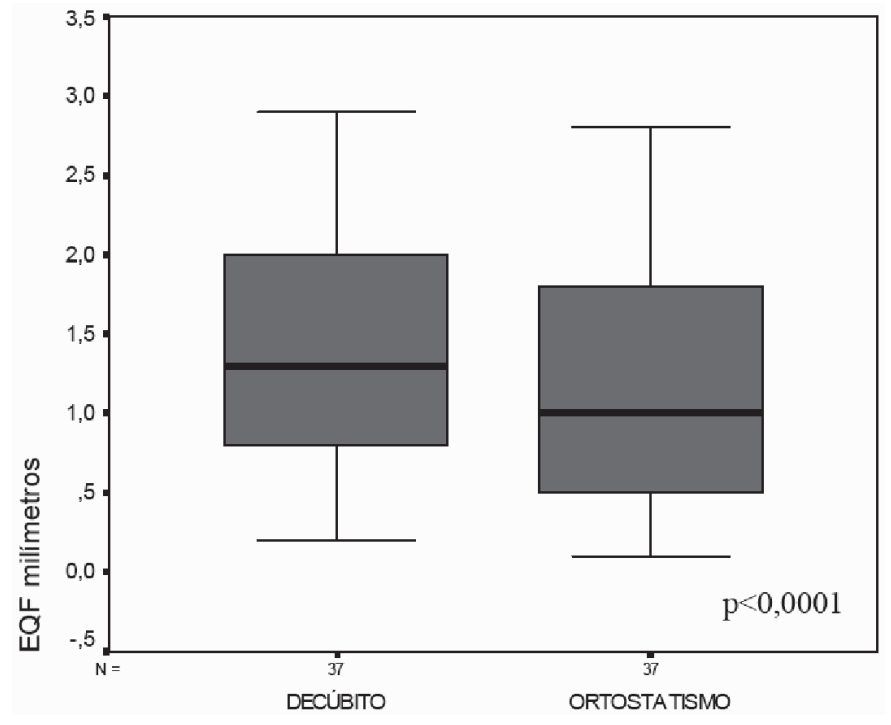

Gráfico 1 - Observar a explícita diferença de alteração do espaço articular nas posições de decúbito dorsal e ortostatismo

priedades de resistência da cartilagem a compressão(3). A migração súpero-lateral da cabeça femoral no quadril está associada à progressão mais rápida da artrose. Já o desvio súpero-medial e medial da cabeça femoral estão envolvidos com uma progressão mais lenta da osteoartrose do quadril ${ }^{(3)}$. Uma distância maior do que $1 \mathrm{~mm}$ no espaço articular do quadril é necessária para que se possa avaliar a progressão da $\operatorname{artrose}^{(1)}$. As alterações anuais no espaço articular relativas ao quadril artrósico são de $0,22 \mathrm{~mm}$ a $0,33 \mathrm{~mm}$ ao ano ${ }^{(1)}$.

O coeficiente de variação intra-observador da medição do espaço articular variou de $5 \%$ a $10 \%$, usando réguas milimetradas. $\mathrm{O}$ espaço súpero-lateral é o que apresentou maior reprodutibilidade ${ }^{(3-4,6-7)}$. Critérios de 
magnificação das radiografias e imprecisão intra-observador são questões importantes a serem abordadas. Basicamente, existem dois métodos para mensuração do espaço articular: o sistema computadorizado de leitura radiográfica e o método que usa réguas milimetradas. Nos sistemas computadorizados de leitura radiográfica, o coeficiente de variação intra-observador encontra-se em torno de 3,2\% ${ }^{(3,5-9)}$. O presente estudo avalia a medida do espaço articular do quadril de maneira condizente com a realidade do meio ortopédico brasileiro na questão da avaliação radiográfica.

A diminuição do espaço articular do quadril de 15\% ao longo de um ano pode ser um ponto importante na avaliação da indicação da artroplastia total do quadril. Esse método possui uma sensibilidade que chega a $75 \%{ }^{(10)}$. Estudos apontam que a diminuição de $0,4 \mathrm{~mm}$ do espaço articular pode gerar importantes repercussões clínicas ${ }^{(11-12)}$.

Pode-se observar, pelos resultados obtidos a partir da população estudada, que existe diferença estatisticamente significativa na diminuição do espaço articular na porção súpero-lateral do quadril com osteoartrose, quando comparadas às posições de decúbito dorsal e ortostatismo monopodálico ${ }^{(3,5,7)}$.

\section{CONCLUSÕES}

Comparando-se 74 radiografias de 28 paciente com osteoartrose do quadril obtidas em decúbito dorsal e ortostatismo monopodálico, conclui-se que:

Existe diferença estatisticamente significativa entre as posições estudadas respectivamente de $1,47 \mathrm{~mm}$ para $1,12 \mathrm{~mm}(\mathrm{p}<0,0001)$;

As radiografias mensuradas em ortostatismo com apoio monopodálico avaliam melhor o verdadeiro espaço articular e o grau de artrose.

\section{REFERÊNCIAS}

1. Ravaud P, Ayral X, Dougados M. Radiologic progression of hip and knee osteoarthritis. Osteoarthritis Cartilage. 1999;7(2): 222-9.
2. Altman RD, Fries JF, Bloch DA, Carstens J, Cooke TD, Genant $\mathrm{H}$, et al. Radiographic assessment of progression in osteoarthritis. Arthritis Rheum. 1987;30(11):1214-25.

3. Conrozier T, Lequesne MG, Tron AM, Mathieu P, Berdah L, Vignon E. The effects of position on the radiographic joint space in osteoarthritis of the hip. Osteoarthritis Cartilage. 1997; $5(1): 17-22$

4. Vignon E, Conrozier T, Piperno M, Richard S, Carrillon Y, Fantino O. Radiographic assessment of hip and knee osteoarthritis. Recommendations: recommended guidelines. Osteoarthritis Cartilage. 1999;7(4):434-6.

5. Spector TD, Cooper C. Radiographic assessment of osteoarthritis in population studies: whither Kellgren and Lawrence? Osteoarthritis Cartilage. 1993;1(4):203-6.

6. Theiler R, Stucki G, Schutz R, Hofer H, Seifert B, Tyndall A, et al. Parametric and non-parametric measures in the assessment of knee and hip osteoarthritis: interobserver reliability and correlation with radiology. Osteoarthritis Cartilage. 1996;4(1): $35-42$.

7. Conrozier T, Jousseaume CA, Mathieu P, Tron AM, Caton J, Bejui J, et al. Quantitative measurement of joint space narrowing progression in hip osteoarthritis: a longitudinal retrospective study of patients treated by total hip arthroplasty. Br J Rheumatol. 1998;37(9):961-8.

8. Gunther KP, Sun Y. Reliability of radiographic assessment in hip and knee osteoarthritis. Osteoarthritis Cartilage. 1999;7(2): 239-46.

9. Buckland-Wright. Radiographic assessment of osteoarthritis: comparison between existing methodologies. Osteoarthritis Cartilage. 1999;7(4):430-3.

10. Maillefert JF, Gueguen A, Nguyen M, Berdah L, Lequesne M, Mazières B, et al. Relevant change in radiological progression in patients with hip osteoarthritis. I. Determination using predictive validity for total hip arthroplasty. Rheumatology (Oxford). 2002;41(2):142-7.

11. Maillefert JF, Nguyen M, Gueguen A, Berdah L, Lequesne M, Mazières B, et al. Relevant change in radiological progression in patients with hip osteoarthritis. II. Determination using an expert opinion approach. Rheumatology (Oxford). 2002;41(2): 148-52.

12. Auleley GR, Duche A, Drape JL, Dougados M, Ravaud P. Measurement of joint space width in hip osteoarthritis: influence of joint positioning and radiographic procedure. Rheumatology (Oxford). 2001;40(4):414-19. 